\title{
Cutaneous contact allergy to a glucose monitor
}

\author{
Joel G. DeKoven MD MHSc, Neil H. Shear MD
}

Cite as: CMAJ 2020 March 16;192:E286. doi: 10.1503/cmaj.191216

A

52-year-old woman with type 2 diabetes presented with a history of an itchy rash on her left arm, directly corresponding to where she wore a 24-hour interstitial glucose monitor, the FreeStyle Libre (Figure 1). The rash had occurred 2 months after the patient had started using the device, and it resolved without medical treatment. When she restarted the FreeStyle Libre on her right arm, it caused a similar reaction, prompting her to discontinue its use. Our differential diagnosis included irritant and allergic contact dermatitis.

Patch testing with the North American Contact Dermatitis Group screening series and metal and (meth)acrylate series showed a sole vesicular positive reaction to isobornyl acrylate at 7 days, confirming a diagnosis of allergic contact dermatitis. Isobornyl acrylate has been isolated by gas chromatography-mass spectroscopy as the responsible allergen in the FreeStyle Libre device. ${ }^{1}$ This acrylic monomer was identified in the plastic shell around the needle of the sensor set, with subsequent migration into the skin adhesive. Causative allergens associated with other brands of glucose monitors include ethyl cyanoacrylate, known for its use in fast-acting "super glues," and colophony, widely used in adhesives. $^{2}$

Interstitial glucose monitors have become increasingly popular, as there is no need for pinpricking to monitor glucose levels. The incidence of allergic contact dermatitis related to the FreeStyle Libre device has been estimated at $0.7 \% .^{2} \mathrm{~A}$ small subgroup of patients has managed the skin allergy by inserting protective pads, and others have switched to an alternative device. ${ }^{3,4}$ Physicians may wish to consider patch testing for patients who experience skin reactions to their glucose monitor.

\section{References}

1. Herman A, Aerts $\mathrm{O}$, Baeck M, et al. Allergic contact dermatitis caused by isobornyl acrylate in Freestyle ${ }^{\circledR}$ Libre, a newly introduced glucose sensor. Contact Dermatitis 2017;77:367-73.

2. Hyry HS, Lippi J, Virtanen H. Allergic contact dermatitis caused by glucose sensors in type 1 diabetes patients. Contact Dermatitis 2019;81:161-6.
3. Kamann S, Oppel E. Hydrocolloid blister plaster decreases allergic contact dermatitis caused by Freestyle Libre and isobornyl acrylate. Contact Dermatitis 2019;81:380-1.

4. Oppel E, Kamann S, Reichl FX, et al. The Dexcom glucose monitoring system an isobornyl acrylate free alternative for diabetes patients. Contact Dermat 2019;81:32-6.

\section{Competing interests: None declared.}

This article has been peer reviewed.

The authors have obtained patient consent.

Affiliation: Division of Dermatology, Department of Medicine, Sunnybrook Health Sciences Centre and the University of Toronto, Toronto, Ont.

Correspondence to: Joel DeKoven, joel.dekoven@sunnybrook.ca

Clinical images are chosen because they are particularly intriguing, classic or dramatic. Submissions of clear, appropriately labelled highresolution images must be accompanied by a figure caption. A brief explanation (300 words maximum) of the educational importance of the images with minimal references is required. The patient's written consent for publication must be obtained before submission. 